the patient. Further, if the case on perineal section had seemed to be one of pure cystitis, then the section could have been utilized as a remedial measure, as suggested by Dr. Weir, of New York, in the Medical Record for June 12, 1880, page 659 , by giving free and easy drainage to the organ, and a better and more perfect channel for irrigation.

IV. The difficulty of determining from whence the purulent discharge proceeded. I believe that it is not certain that the epithelial cells of the pelvis of the kidney are characteristic, and if they are, they are not always present or detected. Moreover, the urine is not always alkaline in a chronic case of cystitis of a high grade.

\section{A CASE OF FACE PRESENTATION WITH SOME REMARKS ON THE TREATMENT OF THIS CLASS OF CASES. 1}

\section{BY J. B. SWIFT, M. D.}

AT a meeting of this Section last year Dr. Harlow reported a series of twelve cases of face presentation, and showed that the labor might be easier for the mother and safer for the child by an early rectification of the position.

I have recently had a case which I succeeded in converting from face into a vertex, and which $I$ think may be of interest in exciting a discussion as to the proper treatment of these cases.

On the morning of September 9, 1883, about nine o'clock, I was called to attend Mrs. O'D., and on my arrival found her in labor with her fifth child. She says her previous labors bave always been tedious and slow, and from the last she was a long time in getting up. She has been married seven years, and is of a decidedly nervous temperament.

Her pains began about two A. M., and although not very severe nor frequent she has had no sleep since they began. Examination per vaginam showed the cervix entirely obliterated, the os open so as to admit the finger, the membranes intact, head presenting, and the anterior fontanelle directly over the os: 'The head was movable above the brim and could be easily turned over so as to make O. R. P., but it would not remain so. The patient was dressed and about the ronm, and had eaten her breakfast as usual.

At eleven A. M. there had been no change, and she had had no pains since the first examination. She was advised to lie down and try to get some sleep, taking, if necessary, one drachm of bromidia, which had been prescribed by her regular attendant, and found to act well at times when she suffered from insomnia.

At two P. M. I found thatshe had slept somewhat without the aid of the medicine, and that the pains had returned, the os being somewhat more dilated than at the previous examination. The head remained in the same position.

At three o'clock I found the os about three quarters dilated and the pains changing in character, becoming expulsive. The head had become more extended, the orbit now occupying a position in the centre of the superior strait. The head was still movable, but pressure on the forehead would now only turn the head so as to

1 Read before the Section for Obstetrics and Gynæcology of the Suffolk District Medical Society, November 21, 1883. bring the anterior fontanelle into its original position. I intended to wait until the os was more fully dilated and then introducing the hand rupture the membranes and bring down the occiput, hoping that with the escape of the waters the head would remain flexed. Unfortunately with the next pain the membranes ruptured spontaneously, and I found the head fully extended, the nose being in the centre of the strait. Pressure on the chin would still convert it into a brow, but the head would turn no farther. I informed the husband of the condition of affairs, and explained to him that I intended to give the woman ether and try to rectify the position. He asked that Dr. F. Minot, who had seen her in one or two of her previous confinements be called in consultation. On his arrival I told him what I proposed doing, and he agreed to the proposition.

The patient was etherized, and introducing my left hand I grasped the occiput, drawing it down into the pelvic cavity and placing it in the right anterior position.

It was deemed advisable to finish the delivery then, rather than leave it to the natural efforts, so the forceps were applied and a female child was extracted. After the delivery of the placenta there was some hæmorrhage, which was controlled by a hot water douche. The woman made an easy and rapid convalescence, the temperature never going above $99^{\circ} \mathrm{F}$.

We are told in our obstetric books that the older accoucheurs believed spontaneous delivery by the face to be impossible, and that they always terminated such cases by version. Playfair says : "Smellie first recognized the fact that spontaneous delivery is possible, and that the chin turns forward and under the pubes; but it was not until long after his time, and chiefly after the appearance of Mme. Lachapelle's essay on the subject, that the fact that most cases could be naturally delivered was fully admitted aud acted upon."

Such being the case, version was given up and the cases were left to the natural efforts; but it was soon found that these cases were long and tedious, and the prognosis for both mother and child was more unfavorable than in cases where the vertex presented; thus Cazeaux says: "We must remark that, as a general rule, the labor is more tedious, more painful, and more dangerous, both to the mother and child, and that it much oftener demands the intervention of art." According to Winckel the mortality of the children amounted to thirteen per cent., while that of the mothers reached as high as six per cent. Playfair says : "As regards the mother, in the great majority of cases the prognosis is favorable, although the labor is apt to be prolonged, and she is therefore more exposed to the dangers attending tedious delivery. As regards the child, the prognosis is much more unfavorable than in vertex presentations. Even when the anterior rotation of the chin takes place in the natural way it is estimated that one out of ten children is still-born; while if not, the death of the child may almost certainly be expected."

Schroeder says: "The prognosis in face presentations, even with the usual mechanism, is far more unfavorable than in head presentation. While in the latter vearly five per cent. of the children are still-born, that proportion rises to thirteen per cent. in face presentations. For the mother, also, face presentations are more unfavorable than head presentations, because on an average the labor lasts much longer. Of course, the 
prognosis is still more unfavorable if the mechanism is abnormal, that is, when the chin does not take its forward turn. Then scarcely a child will be born alive." And finally Lusk: "Though spontaneous delivery is the rule in face presentations, the dangers to both mother and child are considerably greater than in vertex presentations."

In spite of this unfavorable prognosis we are told to leave these cases to nature, only interfering when it becomes evident that the child cannot be born while in this position. Then, if the head is still movable at the brim, or can be pushed up, there are two methods to be applied: (1) pressure on the chin in such a direction as to cause the head to revolve on its transverse axis; or (2), drawing down the occiput by the hand or vectis introduced into the uterus. These measures failing, or if the head is so firmly fixed in the cavity that it cannot be pushed back, the forceps must be used.

Schatz has proposed a method, by external manipulation, which is to be employed early in the labor. I have not seen the original article, but Lusk speaks of it, though he does not say with what success its employment has been attended. The operation consists in restoring the child to the normal attitude by pressing on the chest in one direction, while with the other hand the breech is pressed in the contrary direction, this having the effect of raising the head and at the same time doubling the child up, when the head will resume its normal position, and the vertex will present. 'There can be no question that, if this can be done, it should be tried in all cases where the diagnosis is made early, and I regret that it was not attempted in the case which has been reported, but had it been attempted I think it somewhat doubtful whether it would have been successful, for, although when the extension of the head was first discovered it could be corrected by pressure on the forehead, it would not remain so when the pressure was removed. So in any case, although we might be able by this method to remedy the malposition, still the causes which acted originally in making the face present probably would remain, and there is no certainty that the head would stay in its proper position if put there.

The question then arises whether or not in such cases we are justified in going still farther. It seems to me that we are, especially when we take into consideration the unfavorable proguosis to both mother and child, and the fact that it is much easier to rectify all malpositions early than when labor has been going on for some time. Still I would not lay this down as an absolute rule to be followed in all cases, but my plan of treatment would be in all cases to attempt to rectify the position by Schatz's method. If successful, well and good; but if the malposition returns after being rectified, or not being successful in our attempts at rectification, watch the dilatation of the os closely, care being taken not to rupture the membranes; and when the ${ }^{\prime}$ ' is sufficiently dilated correct the position by pressure on the forehead, rupture the membranes, and keep up the pressure until the pains have caused the vertex to engage in the brim, when the case, if otherwise all,right, can be left to nature. Or if on our first examination we find the membranes ruptured and the head still above the brim, try to correct it by pressure in the manner already described, or, not heing able to do this, introduce the hand, grasp the occiput, and bring it down. There are cases, no doubt, in which the pelvis is roomy or the head small, where the face will engage easily and come through the pelvis without much more effort than in a great many vertex cases, but I think it will be found in the large majority of face cases that this is not the rule. The dimensions of the presenting part cannot be reduced in the way they are when the vertex presents, for the bones of the face are united, and will not overlap as the parietals will, therefore a greater exertion is required to force the face through, thus causing a tedious and harder labor, with the risk of tiring out the patient. Another thing to be thought of is the appearance which the child presents after being born in this way. The swelling known as the caput succedaneum of course forms on the face, and Playfair says of this : "Even when the child is born alive the face is almost always greatly swollen and disfigured. In some cases the deformity produced in this way is excessive, and the features are often scarcely recognizable." In spite of our assurance that this disfiguration will all pass away in a few days it must produce rather an unpleasant sensation, to say the least, to present such a child to a woman who has just passed through such an amount of suffering. Probably no one would object to the employment of Schatz's method.

In speaking of the other methods Lusk says : "Though occasionally successful they have been discountenanced by most obstetric writers, because experience has shown the results to be by no means commensurate with the dangers incurred." But he does not tell us what these dangers are. Further along, however, in speaking of the treatment necessary for cases which have been left to nature, and where she has failed, he shows how much more the danger is increased to the woman, and the child is almost always dead.

Playfair argues against interference on the ground that most men are not skillful enough, and then goes on to say in the next sentence: "When once the face has descended into the pelvis difficulties may arise from two chief causes, - uterine inertia, or non-rotation forwards of the chin." After speaking of the treatment for inertia he continues: "The second class are much more important, and may try the resources of the accoucheur to the utmost." It seems to me that it requires much more skill to handle a case where rotation fails than to merely turn the head over while it is still movable above the brim.

Cazeaux, speaking of the treatment of face cases, says: "In the present state of our science the mento. anterior positions should be left to nature, yet does the same rule hold good with regard to posterior ones? In a word, if this last position be clearly recognized before or shortly after the membranes are ruptured, should we not, prior to the engagement of the face, and while the head is still movable, endeavor to convert it into a vertex position, and thus prevent the difficulties that might subsequently arise? If I had to decide under such circumstances $I$ would certainly resolve the question in the affirmative." Has our science advanced sufficiently since his time to warrant us in our undertaking? It seems to me that it has. The great objection in my mind to interfering with cases where the chin is anterior would be that they would be converted into cases with the occiput posterior; but even here, with our present knowledge of the mech. anisın of these cases, it seems that I should prefer a case of occiput posterior rather than one with the face presenting. 